\title{
Analyse de la forme des tiges du cèdre du Maroc Application à la détermination des courbes de profil
}

\author{
O. M'HIRIT et J.G. POSTAIRE * \\ Ecole nationale forestière d'Ingénieurs, B.P. 511, Sale, Maroc \\ * Centre d'Automatique de l'Université de Lille 1, 59655 Villeneuve-d'Ascq Cedex, France \\ Détaché à la Faculté des Sicences, Université Mohamed V, Rabat, Maroc
}

\section{Résumé}

Pour estimer les volumes de bois correspondant à différentes découpes, les forestiers utilisent des courbes de profil qui indiquent le diamètre en tout point de la tige d'un arbre, quelle que soit sa taille. L'estimation du volume des arbres sur pied à partir de la mesure d'un nombre réduit de dimensions nécessite donc une connaissance approfondie de la forme des tiges.

Dans cet article, la forme des tiges est d'abord soumise à une analyse multidimensionnelïe basée sur l'utilisation d'une nouvelle technique de classification automatique. Cette approche permet d'identifier les différents morphotypes résultant de l'action des nombreux facteurs qui influencent la forme des arbres au sein d'un peuplement.

Cette étude montre ensuite, en prenant pour exemple le cèdre du Maroc, comment la construction d'une courbe de profil pour chaque morphotype permet d'améliorer la précision de l'estimation des volumes des arbres sur pied, sans nécessiter pour autant une mesure de la forme des arbres pendant l'inventaire.

Finalement, les résultats obtenus montrent l'intérêt d'utiliser des modèles de profil décrits point par point, sans forme mathématique sous-jacente. Cette représentation nonparamétrique de la forme des tiges, d'emploi très simple et extrêmement souple, est parfaitement adaptée aux contraintes du calcul sur ordinateur.

\section{Introduction}

\subsection{But du travail}

L'analyse de la forme de la tige des arbres est l'un des problèmes fondamentaux de l'étude de la productivité des forêts. En effet, le volume de bois disponible dans une exploitation forestière est non seulement fonction de la taille des arbres, mais également de leur forme (Bouchon, 1974).

Pratique courante de gestion des ressources forestières, l'estimation du volume des arbres sur pied à partir de la mesure de leur hauteur totale $\mathbf{H}$ et de leur diamètre à hauteur d'homme D nécessite une connaissance approfondie de la forme des tiges. En 
effet, seule la courbe représentant les variations du diamètre $d$ d'une tige en fonction de la hauteur $\mathrm{h}$ permet de calculer précisément soit le volume total de la tige, soit le volume de la partie de tige comprise entre deux hauteurs $h_{1}$ et $h_{2}$, soit encore le volume jusqu'à une découpe donnée.

Afin que des arbres de même forme, mais de taille différente, soient représentés par une même courbe, les profils des tiges sont généralement représentés en coordonnées réduites. Les diamètres sont exprimés en pourcentage du diamètre à hauteur d'homme $\mathrm{D}$ et les hauteurs en pourcentage de la hauteur totale H (cf. fig. 1) (LoETsCH et al., 1973; Behre, 1927 ; Cailliez, 1980).

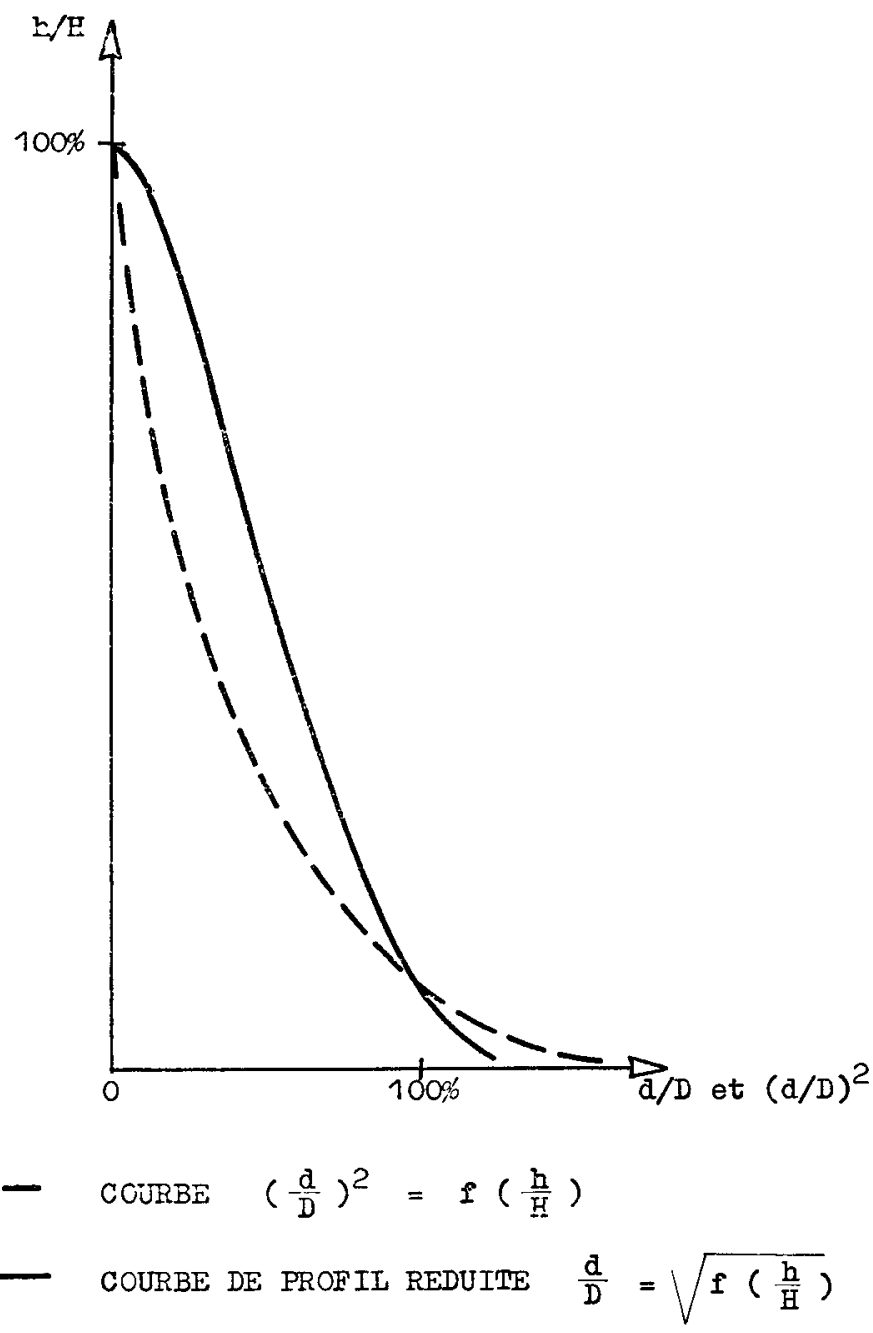

FIG. 1

Représentation du profil des tiges en coordonnées réduites.

Stem profile representation. 
Lorsque la hauteur totale $\mathrm{H}$ et le diamètre à hauteur d'homme $\mathrm{D}$ d'un arbre sont connus, l'intégration de la courbe :

$$
\left(\frac{d}{D}\right)^{2}=f\left(\frac{h}{H}\right)
$$

représentant le carré du diamètre réduit en fonction de la hauteur réduite permet de calculer le volume de la portion de tige comprise entre les hauteurs $h_{1}$ et $h_{2}$ sous la forme :

$$
V=\frac{\pi D^{2} H}{4} \int_{h_{1} / H}^{h_{2} / H} \mathrm{f}\left(\frac{h}{H}\right) d\left(\frac{h}{H}\right)
$$

Si $h_{1}=\mathrm{O}$ et si $h_{2}=H$, on obtient le volume total ce la tige. Lorsqu'on désire connaître le volume jusqu'à une découpe donnée, on applique la formule ci-dessus après avoir déterminé la hauteur de cette découpe à partir de la courbe de profil.

Pour chaque arbre, il est ainsi possible de calculer les volumes de bois correspondant aux différents types d'utilisation : bois d'ébénisterie, bois d'œuvre, bois de trituration, etc. dont les valeurs marchandes sont très différentes.

Le problème est donc d'obtenir la courbe de profil la mieux adaptée au cubage des arbres d'un peuplement, c'est-à-dire celle qui donne les plus faibles erreurs de cubage. L'approche la plus simple consiste à déterminer une courbe de profil représentant la forme moyenne des tiges de l'ensemble forestier étudié. Deux méthodes sont alors envisageables. Historiquement, les premières courbes ont été obtenues en utilisant les mesures effectuées sur les arbres d'un échantillon représentatif pour déterminer graphiquement le diamètre moyen à différentes hauteurs (GERHARDT, 1930 ; Moller, 1933 ; Hummel \& Christie, 1953). Les profils ainsi obtenus, décrits point par point, étaient exploités par des procédures essentiellement graphiques, ce qui explique en partie leur abandon progressif avec l'apparition ces ordinateurs. La méthode la plus utilisée aujourd'hui tire profit des grandes capacités de calcul des machines numériques modernes. Elle consiste à ajuster, par régression, une équation sur les profils mesurés sur les arbres-échantillons (Larson, 1963 ; Fries \& Matern, 1965 ; NASLUnd, 1980 ; PaLm, 1981 ; KozaK et al., 1969). La courbe ainsi obtenue est définie par les valeurs des paramètres figurant dans son expression mathématique. Il s'agit donc d'un modèle paramétrique du profil, qui se prête bien à des manipulations mathématiques telle l'intégration évoquée ci-dessus.

Nous reviendrons, au cours de cet article, sur les mocè̀es définis point par point, sans forme mathématique sous-jacente. Ce type de modèle, essentiellement non-paramétrique, peut en effet, être mémorisé dans un ordinateur, de telle sorte que son exploitation numérique ne pose aucune difficulté particulière. Bien au contraire, nous verrons que la grande souplesse d'utilisation de ce type de modèle doit contribuer à les faire ressortir de l'ombre et leur faire retrouver la place qu'ils ont perdu au profit des modèles paramétriques, souvent très lourds et difficiles à manipuler.

Pour tenir compte de ces fluctuations d'ordre morphometrique, certains auteurs ont caractérisé le profil des tiges par un «quotient de forme», défini comme un rapport 
entre 2 diamètres à des hauteurs différentes (SCHIFFEL, 1905; Jonson, 1910 ; DECOURT, 1965). En divisant la plage de variation de tels facteurs en intervalles égaux, on peut diviser l'éventail des profils rencontrés dans un peuplement en classes plus homogènes et déterminer, pour chacune d'elles, une courbe de profil particulière. La mesure du facteur de forme d'un arbre sur pied permet alors de lui associer la courbe de profil la micux adaptée, ce qui améliore sensiblement la précision de l'estimation des volumes (Behre, 1927 ; Naslund, 1980 ; Baldwin \& Palmer, 1980).

Cependant, cette approche ne présente pas que des avantages. La détermination du facteur de forme des arbres à cuber nécessite une mesure supplémentaire sur le terrain, délicate de surcrô̂t lorsqu'il s’agit d'un diamètre à une hauteur relative (NASLUND, 1980).

\subsection{Principe de la méthode}

Pour pallier les inconvénients liés à l'utilisation des méthodes évoquées ci-dessus, nous proposons, dans cet article, une nouvelle approche au problème de la détermination des courbes de profil et de leur utilisation pour le cubage des arbres sur pied. Cette approche fait interven:- plusieurs courbes de profil pour un même peuplement, afin de prendre en compte les variations de la forme des arbres. Mais alors que la mesure d'un facteur de forme ne permet qu'une division arbitraire des variations de la forme des tiges en un certain nombre de classes, les courbes de profil présentées dans cette étude correspondent à des morphotypes effectivement présent dans les forêts considérées et nettement différenciés. De plus, l'analyse de la forme des tiges n'est entreprise que sur un échantillon réduit, utilisé en partie pour construire les courbes de profil. Sur le terrain les seules mesures nécessaires pour cuber les arbres demeurent le diamètre à hauteur d'homme et la hauteur totale. Cette nouvelle approche, présentée sous son aspect méthodologique, est illustrée par son application au cubage des cèdres du Maroc.

Le principe de base de la méthodologie proposée est de n'envisager la détermination des courbes de profil qu'après une analyse approfondie de la forme des tiges des arbres auxquels l'étude est consacrée. La même démarche a déjà permis aux auteurs d'améliorer sensiblement la précision des tarifs de cubage pour le cèdre du Maroc (M'HIRIT \& Postaire, à paraître). Dans cette étude, comme dans celle consacrée aux tarifs de cubage, il n'est nullement question de réduire la forme d'une tige à un seul paramètre, aussi judicieux soit-il. L'architecture d'une tige résulte d'un ensemble de rapports entre ses dimensions. Elle ne peut donc être décrite et étudiée que par des techniques d'analyse multidimensionnelle.

Pour analyser les variations des caractéristiques dendrométriques des arbres d'un peuplement, on tire des arbres-échantillons dont les tiges sont assimilées à des assemblages simples de 3 troncs de cône (section II - a). Une analyse multidimensionnelle portant sur les 4 paramètres nécessaires pour caractériser ces formes schématiques, après élimination du facteur taille, permet de découvrir les différents morphotypes présents dans la population étudiée (section II - b). C'est ainsi que 3 morphotypes bien différenciés ont été mis en évidence dans 4 grandes cédraies du Rif au Maroc (section II - c).

A chaque morphotype ainsi identifié est associée une forme schématique moyenne. Mais celle-ci est trop éloignée des profils réels pour être utilisée pour le calcul des volumes. On construit donc, pour chaque morphotype, la courbe de profil correspondante à partir des arbres-échantillons qui lui ont été assignés par l'analyse multidi- 
mensionnelle de la forme des tiges. Deux méthodes sont utilisées, l'une avec ajustement d'une équation conduisant à un modèle paramétrique (section III - a), l'autre avec modèle non-paramétrique, déterminé directement à partir des mesures effectuées sur les arbres-échantillons (section III - b). Pour chaque type de modèle, on détermine ensuite une moyenne pondérée des 3 courbes de profil obtenues. Les coefficients de pondération utilisés sont les fréquences relatives de chacun des morphotypes dans la population. Ces courbes sont finalement utilisées pour cuber des lots de cèdres à différentes hauteurs de découpe. De nombreux tests ont montré que les volumes ainsi estimés sont toujours plus précis que ceux obtenus en utilisant une courbe de profil construite directement à partir d'un échantillon unique, tiré aléatoirement de la population (section III - c).

Finalement, cette étude permet de montrer que les gains de précision les plus importants sont obtenus avec le modèle non paramétrique, qui donne d'excellents résultats tout en étant très simple à utiliser (section IV).

\section{Analyse de la forme des tiges}

\subsection{Schématisation de la forme des tiges}

L'expérience montre que, dans les problèmes de reconnaissance des formes, le fait de retenir de nombreux détails dans la description des formes étudiées n'est pas un gage de succès. Bien au contraire la qualité des résultats de l'analyse des formes dans une population est en général conditionnée par le choix d'un nombre récuit de paramètres décrivant l'architecture d'ensemble de ces formes. C'est dans cet esprit que nous avons assimilé la tige de chaque arbre étudié à un assemblage de 3 troncs de cône de révolution (cf. fig. 2). Le premier représente l'empattement de l'arbre jusqu'à la hauteur d'homme, c'est-à-dire $1,30 \mathrm{~m}$. Le second permet de décrire la tige entre $1,30 \mathrm{~m}$ et la mi-hauteur H/2. Le dernier, enfin, représente la moitié supérieure de la tige.

Afin d'éliminer le facteur taille et d'assurer la reconstitution de la tige sous la forme schématique proposée, nous avons retenu, pour caractériser chaque arbre, les 3 paramètres suivants :

- Le coefficient de décroissance :

$$
\delta=\mathrm{d}_{\mathrm{I} / 2 / \mathrm{D}}
$$

où $\mathrm{d}_{\mathrm{II} / 2}$ est le diamètre à mi-hauteur.

- Le coefficient d'empattement :

$$
\varepsilon=\mathrm{D} / \mathrm{d}_{\mathrm{s}}
$$

où $d_{s}$ est le diamètre de la souche.

- L’angle de défilement :

$$
\theta=\operatorname{Arctg}\left(\frac{\mathrm{D}-\mathrm{d}_{\mathrm{H} / 2}}{\mathrm{H}-2,6}\right) \text { (en radians), }
$$

dont la tangente caractérise la décroissance métrique sur le diamètre depuis la hauteur d'homme jusqu'au milieu de la tige. 
Pour nuancer cette schématisation de la forme, nous adjoindrons à ces 3 paramètres le coefficient de la forme $f$ défini par la relation (PARdé, 1961) :

$$
\mathrm{f}=\frac{4 \mathrm{v}}{\pi \cdot \mathrm{D}^{2} \mathrm{H}}
$$

où $\mathrm{v}$ est le volume exact de la tige qui peut être déterminé par la méthode de Huber à partir du cubage de billons successifs (Bouchon, 1974).

Notons que les 4 paramètres qui caractérisent ainsi la forme de chaque tige peuvent être obtenus sans difficulté particulière puisqu'ils ne nécessitent que des mesures couramment effectuées sur le terrain. Cette méthode de caractérisation de la forme des tiges va nous permettre maintenant de procéder à leur analyse fine par l'emploi des techniques de reconnaissance des formes.

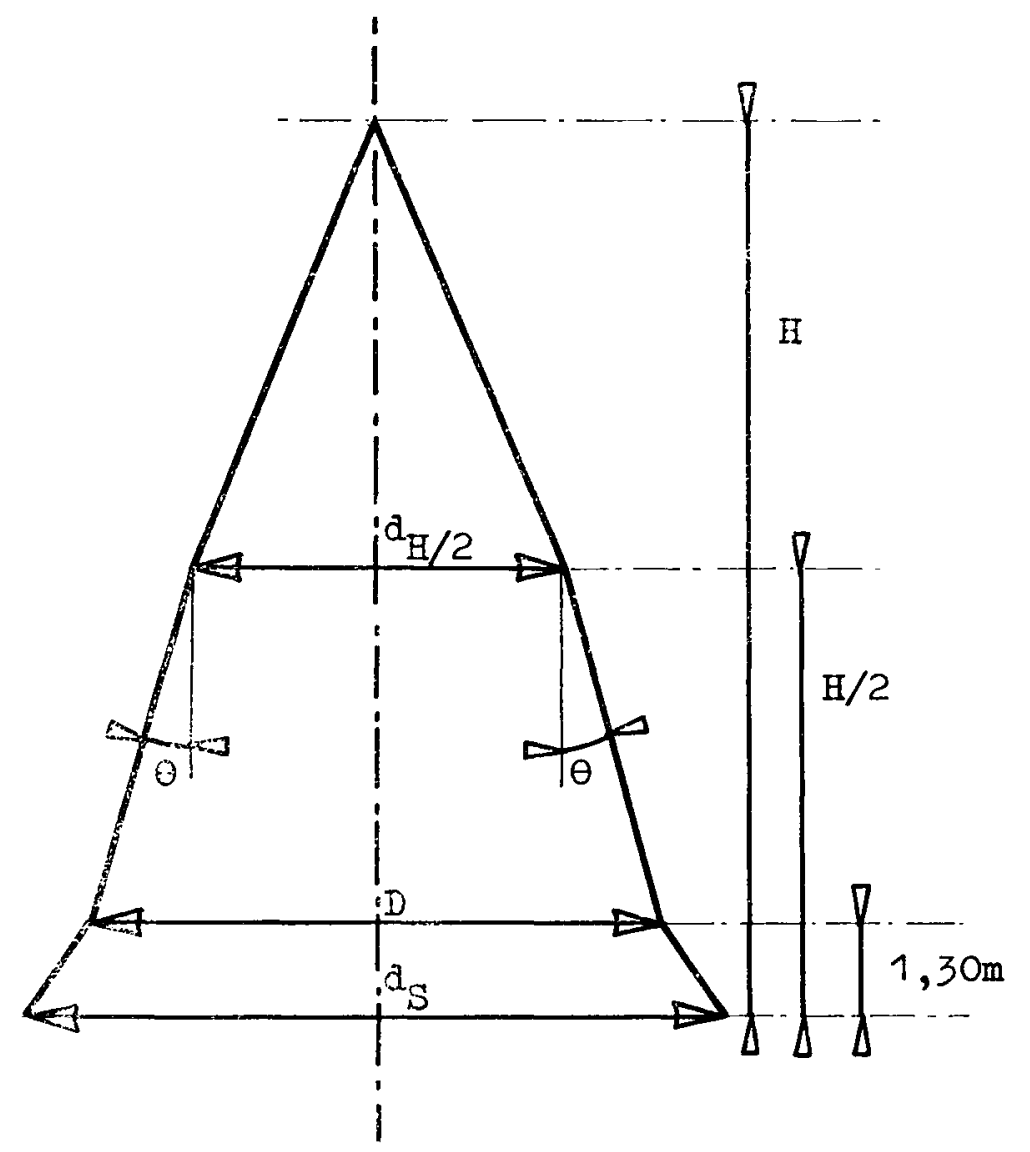

FIG. 2

Schématisation de la forme d'une tige par assemblage de 3 troncs de cône de révolution. Stem shape simplified representation. 


\subsection{Méthode d'analyse de la forme des profils}

Supposons que l'on dispose d'un échantillon constitué d'arbres tirés aléatoirement d'un peuplement et qu'à la forme de la tige de chaque arbre on associe l'observation quadridimensionnelle constituée par les 4 paramètres définis précédemment. La forme de chaque tige peut alors être représentée par un point dans un espace à 4 dimensions.

Cette caractérisation de la forme des tiges permet d'aborder l'analyse morphométrique des arbres par des méthodes d'analyse multidimensionnelle. En effet, l'application des techniques de classification automatique à ces observations quadridimensionnelles doit permettre d'établir, si elles existent, la présence de différentes classes au se:n des échantillons extraits des forêts étudiées, chaque classe correspondant à un morphotype particulier.

Une nouvelle méthode de classification automatique, récemment mise au point par l'un des auteurs, permet d'identifier toutes les classes en présence dans un ensemble d'observations multidimensionnelles, sans aucune information a priori sur les données analysées. De plus, cette méthode détermine automatiquement les caractéristiques d'un élément représentatif de chaque classe mise en évidence et indique la fréquencc relative de lcurs éléments dans la population totale. Finalement, la procédure fournit une classification optimale des observations entre les différentes classes détectées, en ce sens qu'aucune autre stratégie ne peut donner un taux d'erreur de classification plus petit.

Cette méthode et son application aux problèmes de reconnaissance des formes, notamment en foresteric, sont exposées en détail par ailleurs (PostaIrE, 1981 ; PosTaire \& Vasseur, 1981 ; M'hirit \& Postaire, à paraître).

Nous allons maintenant montrer comment cette méîhode permet d'aborder l'analyse morphométrique des cèdres dans quelques massifs forestiers du Rif au Maroc.

\subsection{Polymorphisme du cèdre du Maroc}

Les cèdres étudiés proviennent de 4 grands massifs forestiers du Rif, au nord du Maroc. Les profils des tiges de plus de 1000 cèdres, abattus lors de coupes de regénération et d'éclaircie, ont été mesurés avec précision en notant les diamètres tous les mètres jusqu’à une hauteur de 5 mètres et ensuite tous les 2 mètres jusqu'au sommet des arbres. Les tiges ont également été cubées par la méthode de Hubcr et ces mesures ont été complétées par un relevé précis de leur hauteur totale $\mathrm{H}$ et de leur diamètre à hauteur d'homme D.

La répartition des cèdres entre les 4 massifs forestiers est indiquée au tableau 1. Les résultats d'une étude préalable sur ces arbres (M'HIRIT \& Postaire, à paraître), qui montrent que les profils des cèdres de ces 4 forêts présentent des propriétés morphométriques très voisines, permettent de regrouper les arbres considérés en un échantillon unique. On dispose ainsi, pour l'ensemble des 4 forêts, d'un échantillon d'observations quadridimensionnelles, chaque observation représentant le profil de la tige d'un cèdre. L'analyse de cet échantillon par la méthode de classification automatique évoquée précéciemment a permis de mettre en évidence un polymorphisme très marqué chez les cèdres étudiés. En effet, 3 classes distinctes ont été détectées dans l'échantillon, les arbres assignés à chacune d'elles présentant le même morphotype, c'est-à-dire le 
même type de profil. Les profils moyens caractérisant chaque classe sont représentés sur la figure 3 qui indique également la fréquence dapparition de chaque morphotype dans l'échantillon.
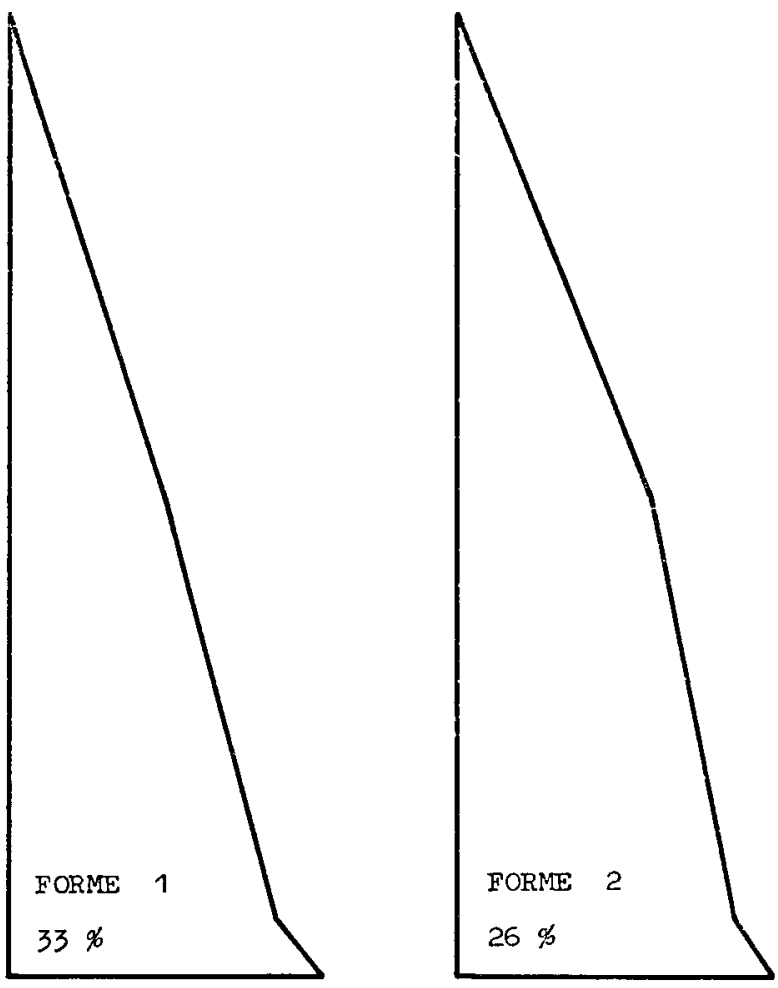

FIG. 3

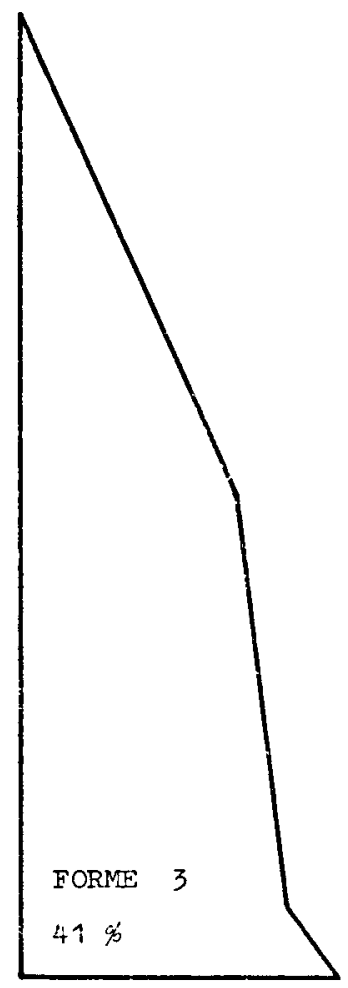

Profils moyens des 3 morphotypes mis en évidence dans les forêts étudiées avec leurs fréquences d'apparition relatives.

Mean stem profile of the three types of form identified in the cedar forests whith their relative frequence.

Les cèdres du Rif présentent donc un polymorphisme naturel dont l'interprétation sort du cadre de cette étude consacrée à la construction de courbes de profil pour ces arbres. La schématisation de l'architecture des tiges par 3 troncs de cône a permis d'étudier les variations de la forme des cèdres et de répartir les arbres-échantillons tirés des forêts en 3 classes. Chacune d'elles constitue en fait un échantillon représentatif d'un morphotype et peut être utilisée pour construire la courbe de profil caractéristique de ce morphotype. 
'I'ABLEAU 1

\begin{tabular}{|c|c|c|c|}
\hline $\begin{array}{c}\text { Nom } \\
\text { du massif forestier }\end{array}$ & $\begin{array}{l}\text { Superficie } \\
\text { (ha) }\end{array}$ & $\begin{array}{l}\text { Altitude } \\
\text { moyenne } \\
(\mathrm{m})\end{array}$ & $\begin{array}{c}\text { Taille de l'échantillon } \\
\text { disponible }\end{array}$ \\
\hline Ghommara & 2320 & 1. 650 & 15.4 \\
\hline Tidighine & 1850 & 2000 & 432 \\
\hline Beni Khennous & 1200 & 2000 & 356 \\
\hline Jbel Lerz $\ldots \ldots \ldots \ldots \ldots$ & 2400 & 1850 & 171 \\
\hline
\end{tabular}

\section{Construction des courbes de profil}

\subsection{Modèle paramétrique}

De très nombreuses études ont été consacrées au choix d'expressions mathématiques appropriées pour représenter le profil des arbres. Devant les difficultés rencontrées pour traduire une forme par une formule mathématique, certains auteurs ont proposé des équations de type polynomial de degré extrêmement élevé (Fries \& MATERN, 1965). D'autres ont proposé de diviser la tige en tronçons pour ajuster un modèle à chacun d'eux en imposant des contraintes pour le raccordement des courbes (Palm, 1981).

Notre propos n'est pas de présenter un nouveau modèle mathématique, mais seulement de disposer d'une référence pour justifier l'emploi des modèles non-paramétriques. C'est pourquoi nous adopterons le modèle parabolique classique et simple :

$$
\left(\frac{d}{D}\right)^{2}=a+b \frac{h}{H}+c\left(\frac{h}{H}\right)^{2}
$$

en lui imposant la contrainte :

$$
\mathrm{a}=-\mathrm{b}-\mathrm{c}
$$

de telle sorte que le diamètre d s'annule au sommet de la tige (KozaK \& Smith, 1966).

Ce modèle, qui se simplifie sous la forme :

$$
\left(\frac{\mathrm{d}}{\mathrm{D}}\right)^{2}=\mathrm{b}\left(\frac{\mathrm{h}}{\mathrm{H}}-1\right)+\mathrm{c}\left(\frac{\mathrm{h}^{2}}{\mathrm{H}^{2}}-1\right)
$$

a été utilisé avec succès pour 17 espèces du Canada (KozAK et al., 1969).

Afin d'obtenir la courbe de profil de chaque morphotype, 50 arbres ont été tirés au hasard parmi les éléments de chacune des 3 classes résultant de la classification de l'échantillon complet tiré de l'ensemble des 4 forêts. L'équation (1) a alors été ajustée par régression sur les profils des 50 tiges de chacun des trois échantillons ainsi constitués.

Les 3 courbes de profil ainsi obtenues sont représentées sur la figure 4 sur laquelle on distingue un profil à tendance conique (forme 1), un profil à tendance para- 
boloïdique (forme 2) et finalement un troisième profil à tendance cylindrique (forme 3 ). On notera que les plus grandes différences de forme de ces profils apparaissent dans la moitié supérieure de la tige. Ces 3 courbes de profil peuvent être comparées à une courbe notée $\mathrm{C}_{\mathrm{p} 1}$, obtenue de manière conventionnelle à partir d'arbres tirés aléatoirement des forêts considérées, sans distinction de forme.

L'intérêt d'avoir un modèle paramétrique pour définir ces courbes de profil est de permettre facilement le calcul du volume de la portion de tige comprise entre 2 hauteurs $h_{1}$ et $h_{2}$ par intégration d'une fonction analytique. Mais, comme nous allons le montrer maintenant, cet avantage n'est pas déterminant car les méthodes d'intégration numérique des courbes définies point par point permettent de calculer ce volume également pour les modèles de profil non-paramétriques.

\subsection{Modèle non-paramétrique}

Historiquement, nous l'avons vu, les premiers modèles de profil n'avaient pas de forme mathématique particulière. Il s'agissait essentiellement de graphiques qui représentaient l'allure moyenne d'un certain nombre de profils réels. La méthode laissait une place importante à l'appréciation du dendrométricien.

Aujourd'hui, il est possible d'obtenir une description point par point des profils, c'est-à-cire sans modèle mathématique, à partir d'un traitement numérique des données sur ordinateur. Lorsque le profil est mémorisé, son intégration pour calculer le volume de la tige entre différents niveaux ne présente aucune difficulté particulière.

Le schéma proposé est de calculer, en coordonnées réduites, la valeur moyenne đu diamètre des tiges de l'échantillon à différents niveaux. Lorsque les diamètres des tiges sont mesurés à des hauteurs fixes, les abcisses des points de mesures en coordonnées réduites sont réparties de manière continue sur toute la hauteur des tiges (cf. fig. 5 a). La méthode la plus simple consiste alors à découper la hauteur totale de la tige réduite en intervalles égaux adjacents et de calculer le diamètre moyen des points situés à l'intéricur de chacun d'eux. Chaque diamètre moyen, assigné à la hauteur du milieu de l'intervalle correspondant, définit ainsi un point du profil moyen de l'échantillon (cf. fig. 5 b). L'ensemble de ces points constitue le modèle non-paramétrique de ce profil moyen (cf. fig. $5 \mathrm{c}$ ).

Cette procédure peut être programmée avec des intervalles de largeur ajustable, celle-ci étant d'autant plus petite que l'on désire une description plus fine du profil des arbres de l'échantillon analysé. On prendra soin toutefois de ne pas trop réduire la largeur de ces intervalles, car, comme dans tout problème de discrétisation, le nombre de points situés à l'intérieur de chaque intervalle deviendrait trop petit pour permettre une estimation correcte du diamètre moyen des tiges.

Cette procédure a été utilisée pour construire les courbes de profil des 3 morphotypes mis en évidence chez le cèdre du Rif. Les courbes ont été obtenues à partir des 3 échantillons de 50 arbres, déjà utilisés pour construire les modèles paramétriques des profils de ces morphotypes. La largeur des intervalles a été ajustée de telle sorte que chaque profil réduit soit représenté par 15 points équidistants auxquels viennent s'ajouter le point représentant le diamètre moyen de la souche et celui représentant le sommet de l'arbre. Les 3 courbes de profil ainsi obtenues sont représentées sur la figure 6 sur laquelle on reconnaît à nouveau les tendances conique (forme 1), para- 


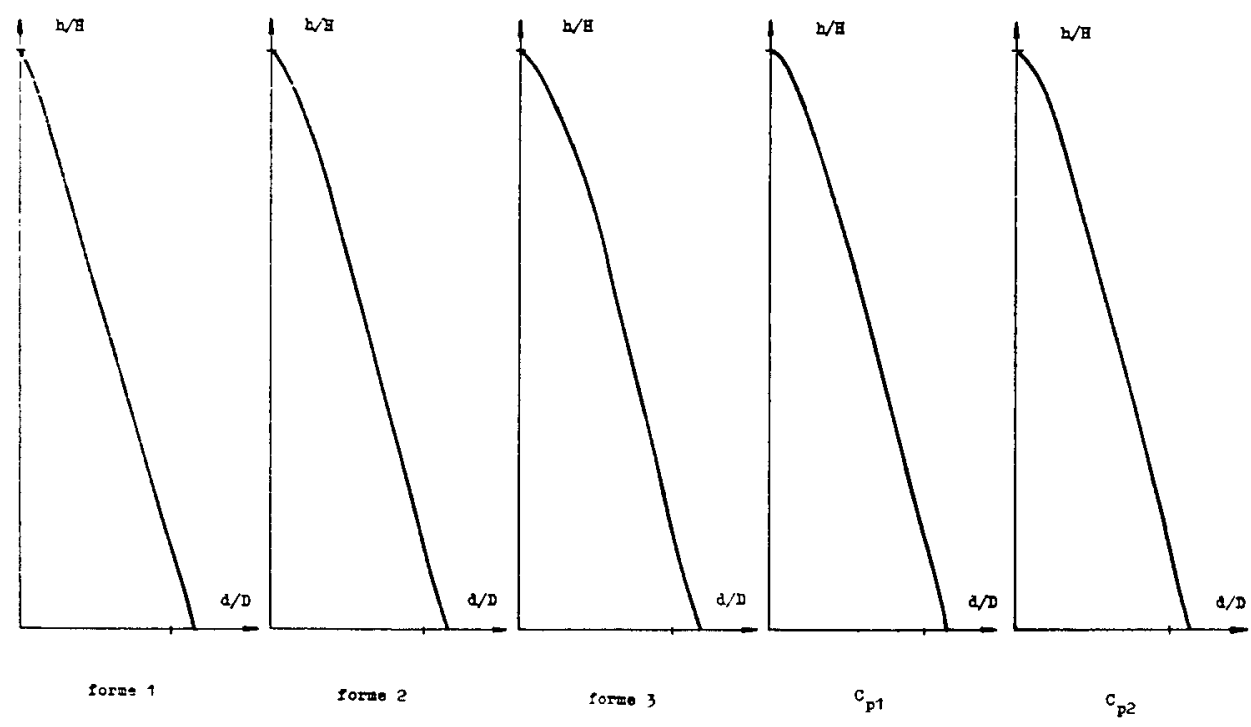

FIG. 4

Courbes de profil paramétriques d'équation :

$$
\frac{d}{D}=\sqrt{a+b \frac{h}{H}+c \frac{h^{2}}{H^{2}}}
$$

Parametric taper curves having equations :

$$
\frac{d}{D}=\sqrt{a+b \frac{h}{H}+c \frac{h^{2}}{H^{2}}}
$$

Forme 1 : Courbe associée au premier morphotype $(a=1,30 ; b=-2,59 ; c=1,29)$. Forme 2 : Courbe associée au deuxième morphotype $(\mathrm{a}=1,28 ; \mathrm{b}=-2,24 ; \mathrm{c}=0,96)$. Forme 3 : Courbe associée au troisième morphotype $(a=1,35 ; b=-2,17 ; c=0,82)$. $C_{m}$ : Courbe obtenue à partir d'un échantillon tiré aléatoirement, sans distinction de forme $(a=1,31 ; b=-2,11 ; c=0,80)$.

$\mathrm{C}_{\mathrm{p}^{2}}$ : Courbe obtenue par moyenne pondérée des courbes associées aux trois morphotypes $(a=1,32 ; b=-2,32 ; c=1,00)$.

Forme 1: Curve associated with the first type of form $(a=1,30 ; b=-2,59$; $c=1,29)$.

Forme 2 : Curve associated with the second type of form $(a=1,28 ; b=-2,24$; $c=0,96)$.

Forme 3: Curve associated with the third type of form $(a=1,35 ; b=-2,17$; $c=0,82)$.

$C_{p_{1}}$ : Curve obtained with simple random sampling $(a=1,31 ; b=-2,11 ; \mathrm{c}=0,80)$.

$C_{p 2}$ : Curve resulting from the weighted mean of the three curves associated to the three types of form $(a=1,32 ; b=-2,32 ; c=1,00)$. 


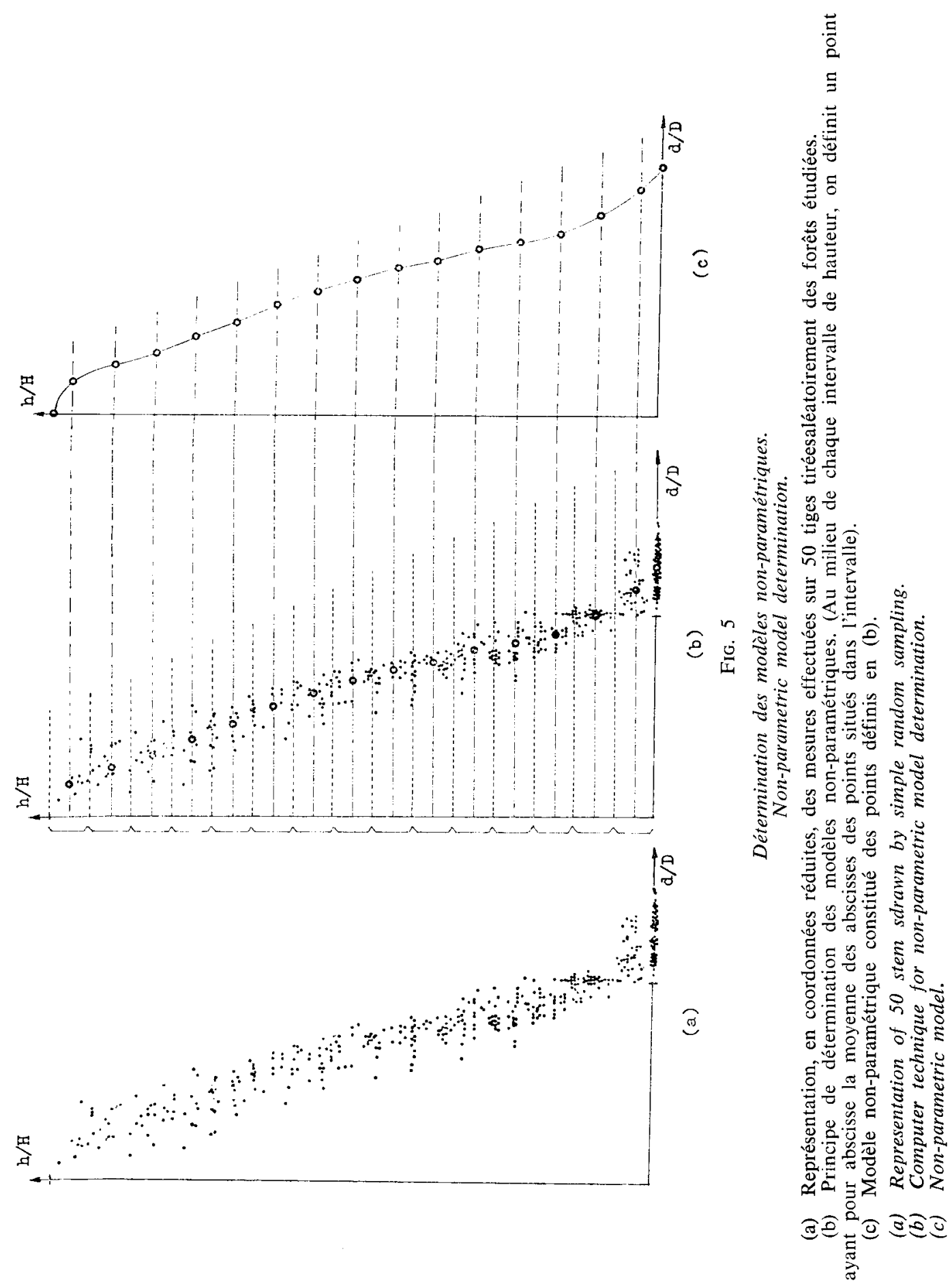


boloïdique (forme 2) et cylindrique (forme 3). Ces 3 courbes peuvent être comparées à une quatrième courbe de profil, notée $C_{n p 1}$, également de type non-paramétrique, mais construite à partir d'arbres tirés aléatoirement des forêts considérées, sans distinction de forme.

L'intégration numérique des courbes :

$$
\left(\frac{\mathrm{d}}{\mathrm{D}}\right)^{2}=\mathrm{f}\left(\frac{\mathrm{h}}{\mathrm{H}}\right)
$$

ainsi définics par 17 points mémorisés dans un ordinateur peut être effectuée par la méthode de Simpson (Bajpal et al., 1975). Cette technique de calcul, d'usage courant en informatique, permet d'aborder $l \mathfrak{c}$ calcul des volumes des tiges à partir des courbes de profil non-paramétrique sans aucune difficulté.

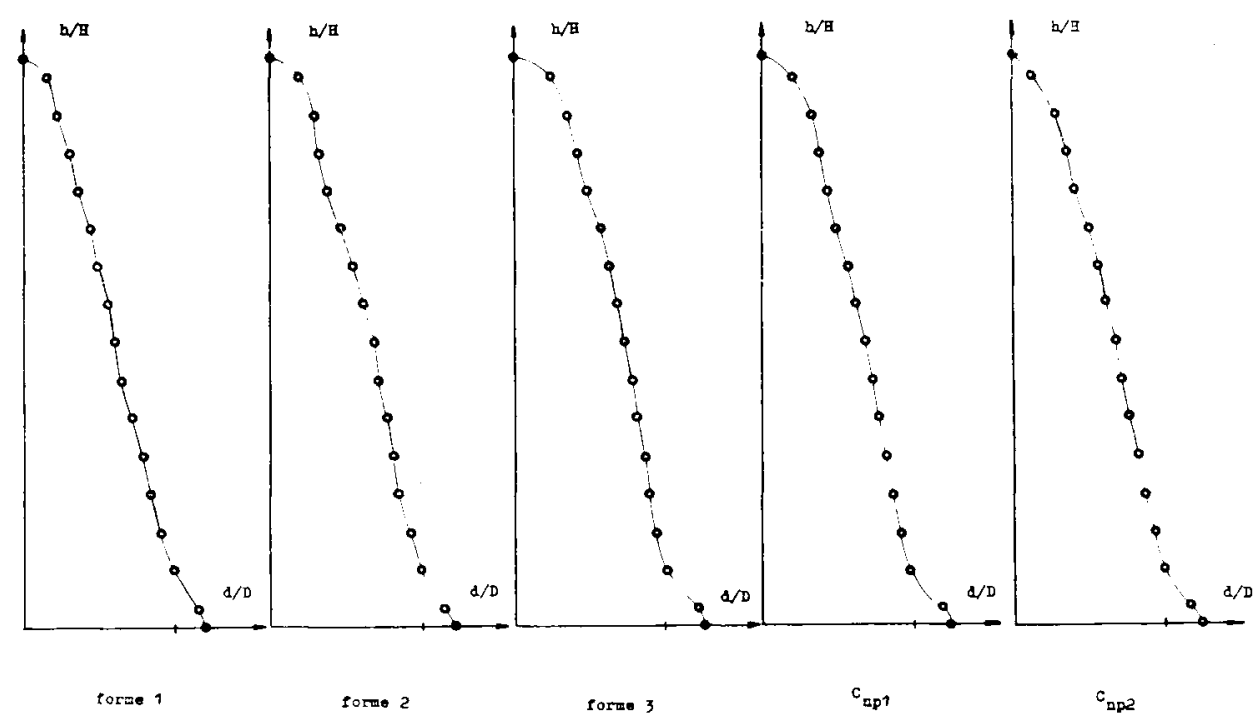

FIG. 6

Courbes de profil non-paramétriques.

Non-parametric taper curves.

Forme 1: Courbe associée au premier morphotype.

Forme 2: Courbe associée au deuxième morphotype.

Forme 3 : Courbe associée au troisième morphotype.

$\mathrm{C}_{\mathrm{nD1} 1}$ : Courbe obtenue à partir d'un échantillon unique, tiré aléatoirement, sans distinction de forme.

$C_{n p 2}:$ Courbe obtenue par moyenne pondérée des courbes associées aux trois morphotypes.

Forme 1: Curve associated with the first type of form.

Forme 2: Curve associated with the second type of form.

Forme 3: Curve associated with the third type of form.

$C_{\mu p r}$ : Cuvre obtained with simple random sampling.

$C_{n p s}:$ Curve resulting from the weighted mean of the three curves associated to the three types of form. 


\subsection{Estimation des volumes à partir des courbes de profil}

Il est évidemment hors de question d'envisager une reconnaissance du morphotype des arbres sur pied au cours des inventaires. Les connaissances approfondies acquises sur la morphologie des arbres étudiés peuvent cependant être exploitées pour améliorer la précision de l'estimation des volumes des tiges.

Pour chaque type de modèle, on définit une cinquième courbe de profil, obtenue en pondérant les 3 courbes correspondant aux 3 morphotypes mis en évidence, les coefficients de pondération étant les fréquences relatives de ces morphotypes dans la population totale (cf. fig. 4 et 6 ).

On dispose ainsi de 4 courbes concurrentes pour représenter le profil moyen des arbres des cédraies étudiées. Pour les modèles paramétriques, une courbe, notée $\mathrm{Cp}_{1}$, est obtenue directement à partir d'un échantillon tiré aléatoirement des forêts, sans distinction de forme. L'autre courbe, notée $\mathrm{C}_{\mathrm{n} 2}$, est une pondération des courbes associées à chaque morphotype. Pour les modèles non-paramétriques, la courbe notée $C_{n p 1}$ est également obtenue sans tenir compte du polymorphisme des cèdres, alors que la courbe notée $\mathrm{C}_{\mathrm{np2}}$ résulte d'une pondération des courbes non-paramétriques représentatives des trois morphotypes.

Afin de comparer leur représentativité vis-à-vis de la forme réelle des arbres, chacune de ces 4 courbes a été utilisée pour établir 2 tables de cubage, l'une donnant le volume de la tige de la souche jusqu'à une hauteur de 5 mètres, l'autre jusqu'à une hauteur de 10 mètres (cf. annexe).

Ces tables ont ensuite été utilisées pour cuber 4 lots de 100 arbres tirés aléatoirement des cédraies du Rif. Suivant une méthodologie déjà éprouvée (M'HIRIT \& PosTAIRE, à paraître), nous avons déterminé, pour chaque lot, la moyenne relative des erreurs de cubage :

avec :

$$
\overline{\mathrm{e}}=\sum_{\mathrm{i}=1}^{100} \mathrm{e}_{\mathrm{i}} / 100 \overrightarrow{\mathrm{V}}
$$

$$
e_{i}=v_{i}-\hat{v}_{i}, i=1,2, \ldots, 100
$$

où $\hat{v}_{i}$ et $v_{i}$ sont respectivement le volume estimé et le volume réel déterminé par la méthode de Huber du ième arbre du lot jusqu'à la hauteur de découpe. $\bar{v}$ désigne le volume moyen exact des tiges du lot considéré jusqu'à cette hauteur.

Nous avons également calculé l'écart-type relatif de la distribution des erreurs de cubage :

$$
\sigma_{\mathrm{e}}=\sum_{\mathrm{i}=1}^{100}\left(\mathrm{e}_{\mathrm{i}}-\overline{\mathrm{e}}\right)^{2} / 99 \overrightarrow{\mathrm{V}}
$$

Pour chaque courbe de profil utilisée et pour chaque hauteur de découpe, nous avons finalement reporté dans le tableau 2 les moyennes de ces valeurs relatives déterminées sur les 4 lots. 


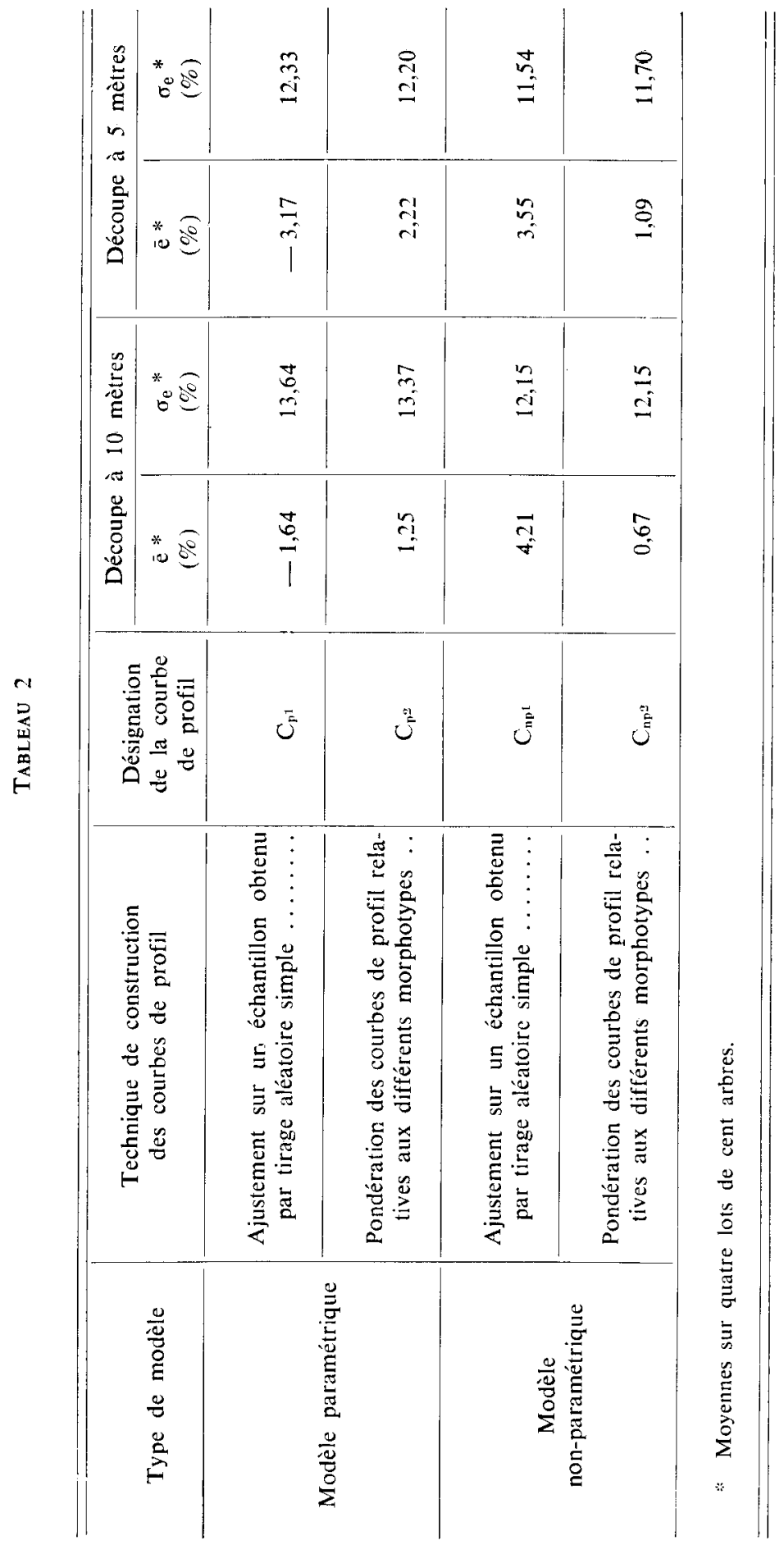




\section{Discussion et conclusion}

Les résultats de lanalyse de la forme des arbres présentés dans cette étude ont incité les auteurs à s'intéresser à la conception d'un instrument efficace pour déterminer, à faible coût les 4 paramètres caractéristiques de la forme des arbres sur pied. En effet, un tel instrument, qui ferait appel à des techniques apparentées à la robotique, permettrait d'utiliser la courbe de profil associée au morphotype de l'arbre considéré. Mais les difficultés techniques rencontrées pour la réalisation de ce type d'appareil risquent d'en retarder l'utilisation en routine sur le terrain. En attendant, et compte tenu de l'impossibilité actuelle de déterminer rapidement et simplement le morphotype d'un arbre sur pied, les résultats de l'analyse de la forme des tiges ont été exploités d'une manière différente. La précision de l'estimation des volumes a été améliorée en définissant des courbes de profil uniques, qui, pour chaque ensemble de forêts considéré, reflètent le mieux possible la répartition des arbres entre les différents morphotypes mis en évidence.

Que l'on utilise des modèles de profil paramétriques ou non-paramétriques, l'examen du tableau 2 montre que la prise en compte du polymorphisme des cèdres étudiés permet d'améliorer sensiblement la précision de l'estimation des volumes des tiges.

En effet, pour chaque type de modèle, les volumes estimés à partir d'un profil obtenu par pondération des 3 courbes associées aux 3 morphotypes sont toujours plus précis que ceux résultant de l'utilisation d'un profil obtenu de manière conventionnelle, sans distinction de forme. Cette amélioration tient sans doute au fait qu'en pondérant les 3 courbes, chaque morphotype contribue au modèle final proportionnellement à sa fréquence relative. Par contre, rien ne garantit que les 3 morphotypes soient représentés dans ces mêmes proportions lorsqu'on tire un échantillon unique et forcément limité pour construire directement un profil moyen.

Il importe également de remarquer que les gains de précision les plus importants ont été obtenus avec les modèles non-paramétriques. C'est en effet ce type de modèle qui permet de représenter au mieux les variations naturelles du diamètre en fonction de la hauteur. Ces variations, qui ne semblent pas toujours soumises à des règles formelles, sont parfois difficiles à modéliser par des fonctions mathématiques. Ces dernières ont en effet tendance à introduire un effet de lissage et à imposer une forme a priori aux modèles des profils. Par contre, le modèle non-paramétrique suit fidèlement les variations du diamètre qui sont ainsi prises en compte dans la courbe obtenue par pondération des profils des 3 morphotypes.

Finalement, il importe de noter que l'utilisation de nos tarifs de cubage ne nécessite, sur le terrain, que la mesure de la hauteur totale $\mathbf{H}$ des arbres et de leur diamètre à hauteur d'homme $D$. Les tarifs présentés ne demandent aucune mesure de paramètre caractéristique de la forme des tiges.

Les résultats de cette étude viennent corroborer ceux obtenus précédemment par les auteurs pour la construction des tarifs de cubage (M'HIRIT \& PosTAIRE, à paraître). L'amélioration des plans d'échantillonnage au moyen d'une stratification par type de forme avait déjà permis d'améliorer sensiblement l'adéquation des équations de volume calculées par régression. Il apparaît maintenant que l'analyse multidimensionnelle de la forme des arbres permet également d'améliorer l'adéquation des courbes de profil. 
Il existe d'autres méthodes de classification automatique, il y a certainement d'autres moyens pour caractériser la forme des tiges, la méthodologie proposée peut être adaptée à d'autres essences forestières : tout un vaste champ d'investigation reste ouvert dont il est encore difficile de prédire les retombées au niveau de l'exploitation des ressources forestières.

$$
\text { Reçu pour publication en décembre } 1982 .
$$

\section{Summary \\ Analysis of the stem form of cedar trees in morocco Application to taper functions determination}

The estimation of stem volume by means of taper functions necessitates a good knowledge of the shape of the trees.

In this paper, a new procedure to determine taper functions is presented. The stem forms of the trees of a forest are first analysed by a multidimensionnal technique which is based on a new classification scheme. The approach allows one to identifie different types of form which are the results of the numerous factors that influence the growth of a tree.

Then, this study shows that the determination of a taper function for each type of form yields an improvement of the precision of volume estimation without requiring any field measurement of form.

Finally, the results demonstrate the advantage in using non-parametric models for taper functions which, also, are well adapted to computer analysis.

\section{Références bibliographiques}

Bajpai A.C., Calus I.H., Fairley J.A., 1975. Numerical methods for engineers and scientists. A student course book, Taylor and Francis, London.

BaldwiN W.C., Polmer B.H., 1980. Taper function for unthinned Longleaf pine plantations on Cutuver-West Gulf sites. Research Conf., Atlanta, Georgia, 156-159.

BEHRE C.E., 1927. Form-class taper curves and volume tables and their application. J. agric. Res., 35, 673-744.

Bouchon J., 1974. Les tarifs de cubage. Publication E.N.G.R.E.F., Nancy, 57 p.

CaIlliez, 1980. Estimation des volumes et accroissements des peuplements forestiers. Vol. 1 : Estimation des volumes. Etude F.A.O. : Forêt 22/1. Rome, Italie.

Decourt N., 1965. Remarques sur la forme et la croissance des tiges. Rev. for. fr., 512-524.

Fries J., MARTEN B., 1965. On the use of multivariate methodes for the construction of tree taper curves. I.U.F.R.O., Advis. Gr. For. Statit., Sect. 25, Conf. Stockholm. Roy. Coll. For., Res. Notes 9, 85-117.

Gerhardt E., 1930. Eine neue (mehrteilige) Buchen-Ertragstafel an Stelle meiner Tafeln von 1909 und 1924. Allg. Forst. Jugdztg, 85 (N.F.), 117-128.

Hummel F.C., Christie J., 1953. Revised yield tables for conifers in Great Britain. For. Rec., Lond., $\mathrm{n}^{\circ} 24$.

JoNson T., 1910. Taxatoriska undersökningar om skogsträdens form. I. granens stamform. Skogsvardsför. Tidskr., 8 (11), 285-328.

Kozak A., Munro D.D., Smith J.H.G., 1969. Taper functions and their application in forest inventory. For. Chron., 45 (4), 278-283. 
Kozak A., Sмith J.H.G., 1966. Critical analysis of multivariate techniques for estimating tree taper suggests that simpler methods are best. For. Chron., 42 (4), 458-463.

LaRSON P.R., 1963. Stem form development of forest trees. For. Sci., Monogr., n ${ }^{\circ} 5$ (Publ. Soc. Am. For.), 42 p.

Loetsch F., Zohrer F., Haller K.E., 1973. Forest inventory. BLV Verlagsgesellschaft, Munich, 469 p.

MOLler C.M., 1933. Boniteringstabeller og bonitetsvise Tilvaekstoversigter for Bog, Eg og Rodgram i Danmark. (Site class tables and yield tables for beech, oak and Norway spruce in Denmark). Dansk Skovforen. Tidsskr., 18, 457-513 et 537-623.

Naslund M., 1980. Stem form studies of pine in northen Sweden. Swedish Univ. Agric. Sci., Rapport $n^{\circ} 8,86 \mathrm{p}$.

Palm R., 1981. Contribution méthodologique au cubage des arbres et à la construction de tables de cubage et d'assortiments. Thèse de Doctorat, Fac. Sci. agron., Gembloux, Belgique, $295 \mathrm{p}$.

PARdÉ J., 1961. Dendrométrie. E.N.E.F., Nancy, 350 p.

Postaire J.-G., 1981. Optimisation du processus de classification automatique par analyse de la convexité des fonctions de densité de probabilité - Application à la reconnaissance de la forme des arbres en dendrométrie. Thèse de Doctorat ès-Sciences, Univ. Lille 1, 220 p.

Postaire J.-G., VAsSeur C., 1981. An approximate solution to normal Mixture identification with application to unsupervised pattern classification. I.E.E.E. Trans. on Pattern Anal. and Machine Intel., PAMI-3, $\mathrm{n}^{\circ}$ 2, 163-179.

Schiffel A., 1905. Form und inhalt des lärche. Mitt. Forstl. Versuchsw. Osterr., 31, 122 p. 